\title{
Simplified Circuit Model for Arrays of Metallic Dipoles Sandwiched Between Dielectric Slabs Under Arbitrary Incidence
}

\author{
María García-Vigueras, Student Member, IEEE, Francisco Mesa, Senior Member, IEEE, \\ Francisco Medina, Fellow, IEEE, Raúl Rodríguez-Berral, and José L. Gómez-Tornero, Member, IEEE
}

\begin{abstract}
This work presents an equivalent circuit to model the transmission/reflection of a plane wave that impinges obliquely on a periodic arrangement of metallic rectangular dipoles embedded between two dielectric slabs. The equivalent circuit takes advantage of the periodicity of the structure to reformulate the original problem as a certain equivalent waveguide scattering problem. Equivalent transmission lines are used to simulate the wave propagation whereas equivalent lumped circuit elements account for presence of the metallic patches. The obtaining of the circuit parameters is carried out via a systematic procedure, which provides a robust strategy that gives rise to surprisingly accurate results even for rather complex situations. The proposed equivalent circuit model simplifies considerably the original complex electromagnetic problem and provides a valuable physical insight into the parameters that are relevant in the phenomenon as well as an in-depth understanding of the operation principles of the periodic surface. Thus, the reported reduced-order model of the corresponding scattering problem can be a very convenient and helpful tool for the analysis and/or design of many practical devices.
\end{abstract}

Index Terms-Equivalent circuits, extraordinary transmission, frequency-selective surfaces (FSSs).

\section{INTRODUCTION}

$\mathbf{F}$ OR many decades, microwave and antenna engineers have dealt with the scattering of electromagnetic plane waves by periodic arrays of metallic patches (or its dual structure, periodic arrays of slots made in a thin metallic surface) [1]-[7]. A similar work was also carried out in the submillimeter and infrared regimes [8]-[11]. More recently,

Manuscript received March 16, 2012; accepted May 01, 2012. Date of publication July 10, 2012; date of current version October 02, 2012. This work was supported by the Spanish Ministerio de Ciencia e Innovación and European Union FEDER funds under Projects TEC2010-16948, TEC2010-21520C04-04, and Consolider EMET CSD2008-00066; by Junta de Andalucía under Project P09-TIC-4595; and by Regional Seneca under Project 08833/PI/08 (Regional Scholarship PMPDI-UPCT-2009).

M. García-Vigueras and J. L. Gómez-Tornero are with the Departamento de las Tecnologías de la Información y las Comunicaciones, Universidad Politécnica de Cartagena, Murcia 30202, Spain (e-mail: maria.garcia@upct.es; josel. gomez@upct.es).

F. Mesa and R. Rodríguez-Berral are with the Microwaves Group, Department of Applied Physics, ETS de Ingeniería Informática, University of Seville, Seville 41012, Spain (e-mail: mesa@us.es; rrberral@us.es).

F. Medina is with the Microwaves Group, Department of Electronics and Electromagnetism, Faculty of Physics, University of Seville, Seville 41012, Spain (e-mail: medina@us.es).

Color versions of one or more of the figures in this paper are available online at http://ieeexplore.ieee.org.

Digital Object Identifier 10.1109/TAP.2012.2207364 paradigmatic examples of the application of periodic distributions of metallic patches, dipoles, or slots can be found in the frame of the so-called frequency-selective surfaces (FSSs) (see, for instance, [12] and [13], and references therein). During the last decade, the interest on periodic structures within the microwave/millimeter wave domains has been focused on the analysis of artificial magnetic conductors, hard and soft electromagnetic surfaces, partially reflecting surfaces, and other structures conceived mainly for antenna applications [14], [15]. Multilayer stacked periodic structures for the implementation of wideband canonical filters [16]-[19] have also received considerable attention during the last few years. In the optical regime, the interest on the behavior of diffraction gratings has a longer tradition. Thus, in-depth studies of this problem can be traced to the seminal works by Wood [20] and Rayleigh [21]. In recent years, the discovery of the phenomenon called extraordinary optical transmission [22] has led to a renewed interest on the modeling of this class of electromagnetic structures; see [23]-[28] and references therein. In spite of some relevant differences among the various frequency regimes due to the different behavior of metals at different frequency regions, all the above problems share a similar physical background. Thus, it is apparent that the accurate modeling of the electromagnetic response of periodic structures made of metal dipoles printed on dielectric substrates (or holes made in a flat conducting surface embedded in a layered dielectric medium) is a topic of great interest for a wide technical and scientific community.

In this paper, we focus on the quasi-analytical modeling of 2-D periodic distributions of rectangular perfect-conductor dipoles or patches in a layered dielectric structure. Apart from the scholarly interest that these periodic sheets may attract, their accurate characterization is essential for many practical applications that make use of their capability of controlling the guidance, reflection, transmission, radiation, and absorption of electromagnetic waves [12]-[19], [29]-[37]. The availability of a quasi-analytical model is always a major advantage from a practical point of view and essential for actual scientific understanding. Thus, any further advance in the analysis of these structures may significantly enhance the modeling and synthesis techniques of many practical devices. Different methods and numerical approaches have been proposed to determine the scattering parameters of these structures. In particular, the equivalent circuit technique has been used for a long time [1]-[3], [6]-[13], [30], [38], [39]. As an interesting and classical example of the application of equivalent-circuit 
methodology, it is worth mentioning that a recently popular problem, the study of the propagation of electromagnetic waves through the so-called wire medium (periodic array of metal wires) [40], [41], was satisfactorily addressed using circuit approaches in the 1950s [42]. More recent examples can be found in [16]-[19], where the equivalent-circuit methodology is employed to understand the behavior of stacked FSS-based filters, or in [31]-[33], where it plays a key role in novel leaky-wave antennas and absorbers.

A very interesting feature of the equivalent-circuit approach is that it reduces the original problem to an equivalent one which consists in the scattering of an incident guided wave by a transverse discontinuity [2], [3] (here, the waveguide is associated with the unit cell of the array). The main advantages of this approach are, on the one hand, that it benefits from the great deal of work carried out previously on waveguide discontinuities [2], [43]-[45] and, on the other hand, that it allows for a rephrasing of the original complex electromagnetic problem in terms of an equivalent circuit network. This last feature means that the potentially complicated wideband frequency response of the system can be accounted for by a small number of parameters. These parameters can eventually be known in closed form for some limited cases but, in general, they should be obtained from just a few full-wave computations. In this way, it should be understood that the equivalent-circuit methodology here proposed is not a substitute of full-wave methods but a very convenient complement that helps to reduce drastically the computational effort. An additional key feature of the equivalent-circuit approach here employed is that it provides a simple and accurate comprehension of the problem. It makes possible many important predictions on the behavior and role of the different elements of the structure under study. This predictive nature can be fundamental for many analysis and/or design applications.

Equivalent-circuit models are commonly limited to specific and narrow frequency ranges. A typical restriction is to consider the unit cell much smaller than the wavelength at the operation frequency. This may not be an important drawback for some applications, but it precludes the use of the model to frequencies where the unit-cell dimensions are close to the wavelength and beyond. However, our proposed circuit model can account for complex high-frequency effects such as extraordinary transmission. (Some of the authors have already reported a circuit-based model [46] that gives a new perspective on the extraordinary transmission phenomenon.) In this paper, the basic guidelines in [46] are adapted to characterize the dual structure: periodic structures formed by arrays of patches or dipoles. However, we now extend significantly the scope of [46] to consider oblique incidence, transverse electric/transverse magnetic (TE/TM) polarizations, and the presence of dielectric slabs. The introduction of oblique incidence as well as dielectric slabs can be carried out in the equivalent-circuit network in a rather straightforward way [13]. Other physical features (losses and other geometrical complexities) could be incorporated to the present circuit model by relatively simple modifications in its topology. Simplified versions of the modeling technique here presented have recently been proposed for 1-D metallic strips gratings (or structures that are reducible to 1-D problems) including dielectric slabs under normal incidence [47]-[50], and a prelim- inary study that this paper considerably extends was reported in [51]. It should be clarified that our proposed circuit model works properly only when applied to single-resonant metallic scatterers. In the derivation of the proposed transverse equivalent network (TEN), it is implicitly assumed that the qualitative shape of the current profile on the scatterers does not change very much in the considered frequency range. To the authors' knowledge, in the case of multiresonant scatterers, the most that can be done is to devise certain ad hoc topology making use of some a priori knowledge of the complete qualitative response of the original structure in the frequency range of interest. In our proposal, we do not use previous information to build the topology of the TEN.

In the remaining sections, we propose appropriate topologies for the equivalent circuit network of a 2-D dipole array, and we discuss a systematic procedure to extract the parameters of the circuit from a few numerically generated full-wave data. The analytical incorporation of the frequency dependence of some of those parameters is a key feature of our procedure. This is crucial to achieve robustness and accuracy over a wide frequency band. The circuit model is valid not only around the resonance frequency of the dipoles or patches, but over a wide frequency band that includes effects such as Wood's anomalies, grating lobes, and extraordinary transmission/reflection (which are not directly linked to the shape and geometry of the scatterers). Our relatively simple equivalent-circuit models can be further combined with full-wave tools, or other sophisticated approaches [30], [37]-[39], to give an accurate analysis/synthesis tool with a low computational cost. Also, the present technique can easily be extended to characterize the complementary structure: metallic plates perforated by arrays of holes, thus complementing [46].

\section{Circuit Model for ARbitrary TE InCidenCE}

In order to present the basic rationale underlying our equivalent-circuit model, we first consider a freestanding periodic surface built from the periodic arrangement of conducting zerothickness metallic patches (dipoles) of dimension $a_{x} \times a_{y}$ (see Fig. 1). The array extends along the $x$ - and $y$-axes, with periodicities $P_{x}$ and $P_{y}$, respectively. The periodic surface is located at $z=0$ and a plane wave impinges on this surface from the direction defined by $\theta$. As can be seen in Fig. 1, the plane of incidence is $x z$ (with azimuthal angle $\varphi=0^{\circ}$ ), and the wave is TE polarized (i.e., its electric field is directed along the $y$-axis). Therefore, in the scanned $H$-plane, the impinging field is always parallel to the metallic dipoles, having a strong interaction with them. Due to periodicity, Floquet (space) harmonics are excited by the incident plane wave as it reaches the array [13], [52]. These scattered waves can be either TE or TM polarized. The wavenumbers in the $x$ - and $y$-axes associated with the space harmonics are given by

$$
\begin{aligned}
k_{x, m} & =k_{0} \sin \theta+\frac{2 m \pi}{P_{x}} \\
k_{y, n} & =\frac{2 n \pi}{P_{y}}
\end{aligned}
$$

where $m, n=0, \pm 1, \pm 2, \ldots$, and $k_{0}=2 \pi f / c$ is the freespace wavenumber, with $f$ being the frequency and $c$ the speed 
of light in vacuum. Each excited space harmonic is defined by a pair of integers $m n$ with the following associated complex wavenumber along the $z$-direction:

$$
\begin{aligned}
& k_{z, m n}^{\left(\varepsilon_{r}\right)} \\
& = \begin{cases}\beta_{m n}=\sqrt{k_{0}^{2} \varepsilon_{r}-k_{x, m}^{2}-k_{y, n}^{2}}, & f \geq f_{c, m n}^{\left(\varepsilon_{r}\right)} \\
-j \alpha_{m n}=-j \sqrt{k_{x, m}^{2}+k_{y, n}^{2}-k_{0}^{2} \varepsilon_{r}}, & f<f_{c, m n}^{\left(\varepsilon_{r}\right)}\end{cases}
\end{aligned}
$$

where $f_{c, m n}^{\left(\varepsilon_{r}\right)}$ is the cutoff frequency of the $m n$th harmonic, namely, the frequency that satisfies

$$
\gamma_{m n}^{2}=k_{x, m}^{2}+k_{y, n}^{2}-k_{0}^{2} \varepsilon_{r}=0
$$

and that can be expressed as

$$
\begin{aligned}
& f_{c, m n}^{\left(\varepsilon_{r}\right)} \\
& =\frac{1}{\varepsilon_{r}-\sin ^{2} \theta} \\
& \quad \times\left\{\frac{m c}{P_{x}} \sin \theta+\sqrt{\varepsilon_{r}\left[\left(\frac{m c}{P_{x}}\right)^{2}+\left(\frac{n c}{P_{y}}\right)^{2}\right]-\left(\frac{n c}{P_{y}} \sin \theta\right)^{2}}\right\} .
\end{aligned}
$$

The above cutoff frequency is determined by the array periodicities $\left(P_{x}\right.$ and $\left.P_{y}\right)$, the angle of incidence $(\theta)$, and the medium dielectric constant $\left(\varepsilon_{r}\right)$, but it is independent on the dipole dimensions. For convenience, the cutoff frequency is here formulated in the presence of a homogeneous dielectric medium with permittivity $\varepsilon_{r}$. This fact is represented by the superscript $\left(\varepsilon_{r}\right)$, although free space is assumed in the present section $\left(\varepsilon_{r}=\right.$ 1 ), which will be denoted by the superscript (1). Note that, for the case of normal incidence $\left(\theta=0^{\circ}\right)$, (5) reduces to the well-known expression of the modal cutoff frequencies in a rectangular waveguide of dimensions $P_{x} \times P_{y}$.

At a given frequency, as (3) reveals, $k_{z, m n}^{(1)}$ can be purely imaginary or real, defining evanescent (slow) or grating (fast) waves, respectively [13]. Specifically, each harmonic is an evanescent wave that decays along the $z$-direction below its cutoff frequency $f_{c, m n}^{(1)}$. Above this frequency, $k_{z, m n}^{(1)}$ is no longer imaginary and the associated harmonic becomes a (grating) plane wave that propagates along a direction parallel to the vector $\mathbf{k}_{m n}=k_{x, m} \hat{\mathbf{x}}+k_{y, n} \hat{\mathbf{y}}+k_{z, m n}^{(1)} \hat{\mathbf{z}}$. In our analysis, the incident plane wave in Fig. 1 corresponds to the dominant $m n=00$ Floquet harmonic. This wave propagates at any given frequency along the direction defined by $\theta$ (note that $k_{z, 00}^{\left(\varepsilon_{r}\right)}$ is always real). In most microwave engineering and antenna applications, the working frequencies are within the so-called no grating lobes regime [13]; i.e., only the dominant Floquet harmonic is propagating while the remaining harmonics are evanescent.

In general, each Floquet harmonic can be either TE or $\mathrm{TM}$ polarized, and has the following associated characteristic impedance [13], [53]:

$$
\begin{aligned}
Z_{\mathrm{TE}, m n}^{\left(\varepsilon_{r}\right)}(\omega) & =\frac{j \omega \mu_{0}}{\gamma_{m n}} \\
Z_{\mathrm{TM}, m n}^{\left(\varepsilon_{r}\right)}(\omega) & =-\frac{j \gamma_{m n}}{\omega \varepsilon_{0} \varepsilon_{r}} .
\end{aligned}
$$

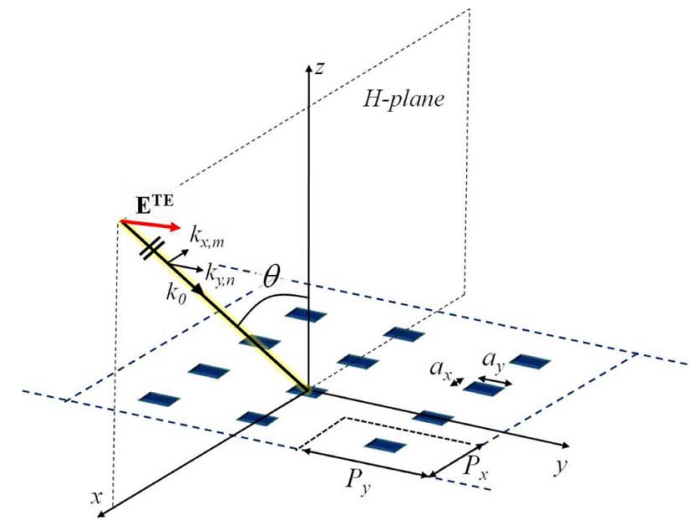

Fig. 1. Scheme of the freestanding array of metallic dipoles of zero thickness under oblique incidence in $H$-plane $\left(P_{x}=P_{y}=5 \mathrm{~mm}, a_{x}=0.5 \mathrm{~mm}\right)$.

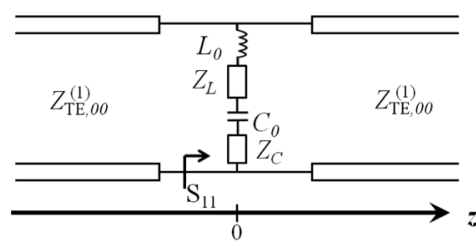

(a)

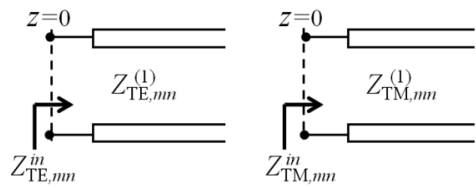

(b)

Fig. 2. (a) Proposed TEN for Fig. 1. (b) Transmission line circuits involved in the definition of $Z_{L}$ and $Z_{C}$.

The above expressions tell us that, depending on the propagative or evanescent nature of the harmonic, its associated impedance is real or purely imaginary. In particular, inductive/capacitive impedances correspond to TE/TM evanescent waves [13], [53]. Due to the assumed impinging TE polarization and plane of incidence, the unit cell of the problem under consideration can be seen as a transmission line formed by two parallel-to- $x z$-plane electric walls separated by a distance $P_{y}$ and two parallel-to- $y z$ -plane Floquet walls separated by a distance $P_{x}$ (see Fig. 1). It should be noted that the set of excited Floquet harmonics corresponds to the modal solutions of this transmission line. $\mathrm{TM}_{m 0}$ harmonics do not satisfy the specified boundary conditions and are not excited in the structure under consideration.

According to the above discussion, the structure in Fig. 1 is proposed to be modeled by the TEN shown in Fig. 2(a). The propagation of the incident, reflected, and transmitted TE plane wave is modeled by the infinite transmission lines placed at both sides of the discontinuity. The characteristic impedance associated to these lines is real and corresponds to

$$
Z_{\mathrm{TE}, 00}^{\left(\varepsilon_{r}\right)}(\omega)=\frac{\omega \mu_{0}}{k_{z, 00}^{\left(\varepsilon_{r}\right)}}
$$

The lumped elements in the series configuration shown in Fig. 2(a) model the excitation of all other $m n$ harmonics 
excited in the discontinuity [13, Ch. 5]. Specifically, the inductive elements $\left(L_{0}\right.$ and $\left.Z_{L}\right)$ account for the excitation of TE harmonics, whereas the capacitive components $\left(C_{0}\right.$ and $\left.Z_{C}\right)$ account for the TM contribution. The impedances

$$
\begin{aligned}
& Z_{L}(\omega)=\sum_{h=1}^{N_{\mathrm{TE}}} A_{h}^{\mathrm{TE}} Z_{\mathrm{TE}, h}^{\mathrm{in}}(\omega) \\
& Z_{C}(\omega)=\sum_{h=1}^{N_{\mathrm{TM}}} A_{h}^{\mathrm{TM}} Z_{\mathrm{TM}, h}^{\mathrm{in}}(\omega)
\end{aligned}
$$

characterize the excitation of the TE and TM harmonics with lowest cutoff frequencies ( $N_{\mathrm{TE}}$ and $N_{\mathrm{TM}}$, respectively). In these expressions, each considered harmonic $h$ is associated with certain $m n$ pair, and $A_{h}^{\mathrm{TE} / \mathrm{TM}}$ is a factor that accounts for the relative degree of excitation of each TE/TM $h$ th harmonic. In this work, we assume that these latter factors are independent of frequency, a feature that is expected provided that the current profile on the scatterers does not change significantly with frequency. The contribution of each TE/TM wave in (9)-(10) is proportional to the input impedance of its corresponding equivalent transmission line, which here corresponds to an infinite transmission line of impedance $Z_{\mathrm{TE} / \mathrm{TM}, m n}^{(1)}$, as shown in Fig. 2(b). The higher order TE and TM harmonics that were not considered in (9)-(10) are highly evanescent waves whose global effect can be accounted for by means of a frequency-independent inductance $L_{0}$ and capacitance $C_{0}$, respectively. This is possible because $\gamma_{m n}$ in (6) and (7) is practically independent of frequency in this case.

Thus, we propose the following general procedure to model the discontinuity effect for frequencies less than a given frequency $\left(f<f_{u}\right)$.

1) Determine the number of TE and TM harmonics above cutoff at $f=f_{u}$ [this number depends on the angle of incidence, as can be deduced from (5)].

2) Take the values of $N_{\mathrm{TE}}$ and $N_{\mathrm{TM}}$ as one plus the number of TE and TM harmonics above cutoff, respectively.

3) Compute the values of the frequency-independent components $\left(L_{0}\right.$ and $\left.C_{0}\right)$ and the excitation coefficients $\left(A_{h}^{\mathrm{TE} / \mathrm{TM}}\right)$ from a few full-wave values of the reflection coefficient $S_{11}$.

It should be considered that the $S_{11}$ parameter in Fig. 2(a) can be related to the lumped-element parameters in this circuit in the following way:

$$
\begin{array}{r}
j \omega L_{0}+\frac{1}{j \omega C_{0}}+\sum_{h=1}^{N_{\mathrm{TE}}} A_{h}^{\mathrm{TE}} Z_{\mathrm{TE}, h}^{\mathrm{in}}(\omega)+\sum_{h=1}^{N_{\mathrm{TM}}} A_{h}^{\mathrm{TM}} Z_{\mathrm{TM}, h}^{\mathrm{in}}(\omega) \\
=-Z_{\mathrm{TE}, 00}^{(1)}(\omega) \frac{S_{11}(\omega)+1}{2 S_{11}(\omega)}
\end{array}
$$

If the above equation is written for a set of frequency values, we obtain a linear system of equations whose solution is the set of unknown parameters of our equivalent circuit model. The number of full-wave computations of the $S_{11}$ parameter is determined by the number of unknowns, namely, $N_{\mathrm{TE}}+N_{\mathrm{TM}}+2$ (typically no more than four or five for freestanding periodic surfaces). The solution to the system of equations is, in general, sensitive to the chosen set of frequency points. However, according to our numerical experience, very stable and physically meaningful solutions are found if two of the evaluated frequency points are taken in the low-frequency regime (these points mostly influence the characterization of the two frequency-independent parameters). In particular, we have used values of frequencies that are 0.01 and 0.03 times the onset frequency of the grating lobe regime. The rest of frequency points (which have more influence in the characterization of the excitation coefficient) are taken as 0.95 times the onset frequency of the $N_{\mathrm{TM}}$ and $N_{\mathrm{TE}}$ harmonics, respectively. If TM and TE harmonics have the same onset frequency, the respective sampling frequencies are taken as 0.95 and 0.98 times this onset frequency.

Following the Fabry-Pérot condition, every zero-thickness metallic dipole in the array resonates at a frequency where its length is roughly half a wavelength [1] (the fringing fields make the Fabry-Pérot condition only approximate). If this condition occurs at a frequency below the grating lobe regime, the array only presents total reflection at this frequency. However, if the Fabry-Pérot dipole resonance does take place in the grating lobe regime, an extraordinary total reflection is still expected to occur before the onset of the first grating lobe (as was reported in [22] for total transmission in a dual structure). Moreover, the extraordinary reflection can be related to the appearance of Fano resonances [54], i.e., a peak of total reflection/transmission which precedes a very close null of reflection/transmission. Therefore, depending on the length of the dipoles, the periodic surface can present either conventional or extraordinary reflection; or, in other words, either Fabry-Pérot- or Fano-type resonance.

Thus, in order to check the validity of our proposed equivalent circuit, the dipole array described in Fig. 1 with $a_{y}=$ $2 \mathrm{~mm}$ is studied in Fig. 3(a). This figure shows the reflectivity under plane wave oblique incidence $\left(\theta=20^{\circ}\right)$ computed with a full-wave method-of-moments approach [34] and with the proposed TEN of Fig. 2. Fig. 3(a) shows an extraordinary resonance (total reflection $\left|S_{11}\right|=1$ ) at approximately $44.5 \mathrm{GHz}$ (the expected dipole Fabry-Pérot resonance should be around $75 \mathrm{GHz}$ ). Three nulls can also be observed in the reflection coefficient at approximately 44.7, 63.9, and $70 \mathrm{GHz}$. These nulls can be associated with Wood's anomalies that appear at the onset of higher order harmonics [46]. Specifically, at the cutoff frequency of TE harmonics, their associated TE impedance diverges to infinity [see (6)], which causes $Z_{L}$ to behave as an open circuit in Fig. 2. Therefore, perfect impedance matching is experienced by the incident wave $\left(\left|S_{11}\right|=0\right)$ at these TE cutoff frequencies [13], [46].

The above fact is illustrated in Fig. 3(b), where the higher order harmonic cutoff frequencies for the considered array are plotted as a function of the angle of incidence. These frequencies are computed using (5), and are identical for both TE and $\mathrm{TM}$ polarization. As mentioned above, no $\mathrm{TM}_{m 0}$ harmonic can be excited. The squares in Fig. 3(b) point out the onset of the first harmonics when $\theta=20^{\circ}$, which occur at 44.7, 63.9, and 


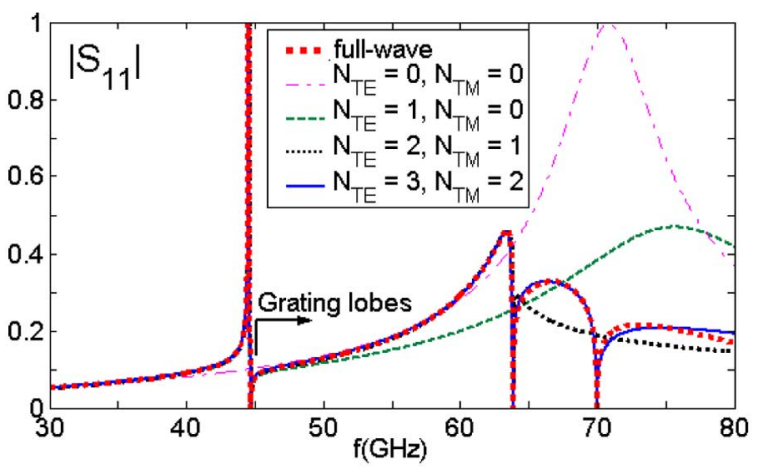

(a)

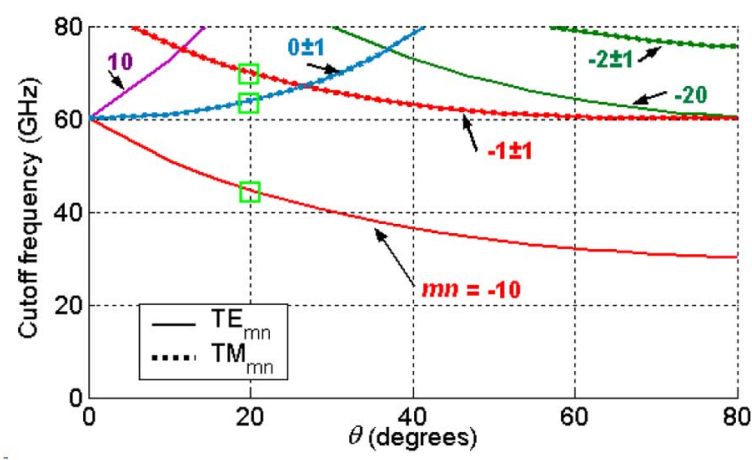

(b)

Fig. 3. (a) Magnitude of the reflection coefficient for the structure in Fig. 1 under $20^{\circ}$ incidence and dipoles with $a_{y}=2 \mathrm{~mm}$. (b) Cutoff frequencies associated with the higher order $m n$th harmonics excited in the structure as a function of the angle of incidence.

$70 \mathrm{GHz}$ (harmonics $m n=-10,0 \pm 1$, and $-1 \pm 1$, respectively) and correspond to the reflectivity nulls in Fig. 3(a). If only the frequency-independent elements $L_{0}$ and $C_{0}$ are considered in the circuit model [curve $N_{\mathrm{TE}}=0, N_{\mathrm{TM}}=0$ in Fig. 3(a)], the array response is accurately predicted only for low frequencies and the aforementioned reflectivity nulls are not obtained. These lumped elements predict a conventional total reflection (Fabry-Pérot-type resonance) at $71 \mathrm{GHz}$. When the impedance of the first higher order TE harmonic is considered in the TEN [curve $N_{\mathrm{TE}}=1, N_{\mathrm{TM}}=0$ in Fig. 3(a)], both the total reflection peak at $44.5 \mathrm{GHz}$ and the first null at $44.7 \mathrm{GHz}$ are accurately obtained. It can then be concluded that the peak at $44.5 \mathrm{GHz}$ is mainly caused by the inductive impedance associated with the first TE higher harmonic in the circuit model $Z_{\mathrm{TE},-10}^{(1)}$. This impedance quickly diverges to infinity close and below the onset of the mode, thus providing the needed inductance to resonate with the capacitance $C_{0}\left(Z_{C}=0\right.$ since $N_{\mathrm{TM}}=0$ ). This resonance makes the equivalent circuit behave as a short circuit, causing the appearance of total reflection $\left(\left|S_{11}\right|=1\right)$. Wood's anomaly associated with the onset of this harmonic (null at $44.7 \mathrm{GHz}$ ) is also accurately predicted. Successive incorporations of the second and third TE harmonics [with respective resonances at 63.9 and $70 \mathrm{GHz}$ in Fig. 3(b)] provide accurate description of the second and third nulls.

It should be noted that the higher order impedances become real after the onset of their corresponding harmonics, resulting in radiation losses in the TEN of Fig. 2(a). The onset of the first

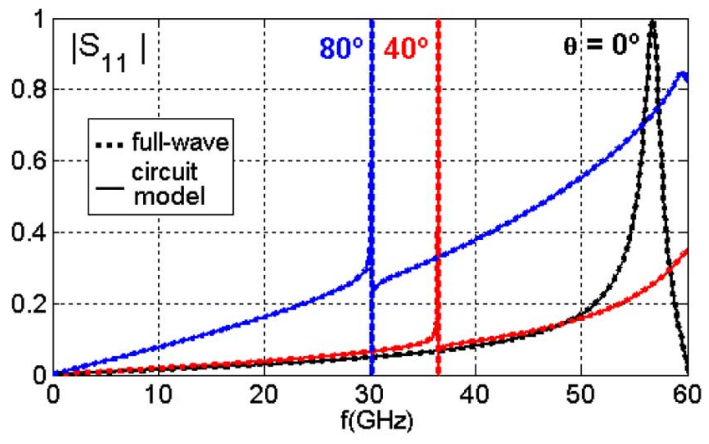

(a)

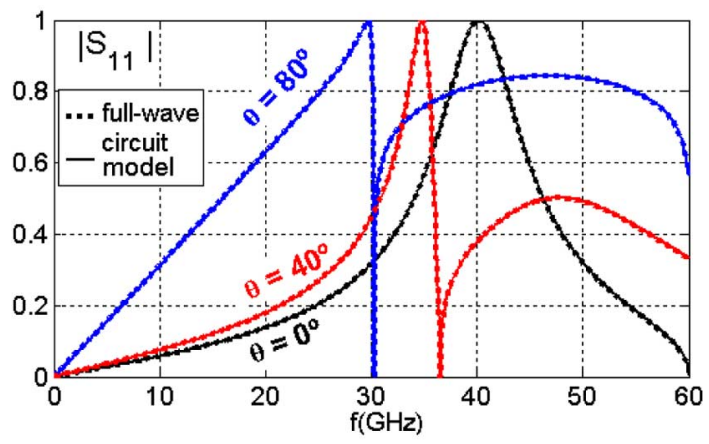

(b)

Fig. 4. Magnitude of the reflection coefficient for the structure in Fig. 1 under different angles of incidence. (a) $a_{y}=2 \mathrm{~mm}$. (b) $a_{y}=3.5 \mathrm{~mm}$.

higher order harmonic determines the beginning of the grating lobe regime. Therefore, above the frequency of the first reflectivity null $(44.7 \mathrm{GHz})$, total reflectivity peaks $\left(\left|S_{11}\right|=1\right.$; i.e., perfect mirror reflectance) cannot occur anymore. Nevertheless, it can be observed in Fig. 3(a) that local maxima appear between every two consecutive nulls. The capacitive impedances associated with TM higher order harmonics are also required to accurately predict the array response. Their inclusion is necessary although they do not produce nulls in the reflection coefficient [since their characteristic impedance does not diverge at their cutoff frequency; see (7)]. In the case treated in Fig. 3(a), the number of higher order harmonics needed to accurately obtain the array response until $f_{u}=70 \mathrm{GHz}$ is $N_{\mathrm{TE}}=3$ and $N_{\mathrm{TM}}=2$. The above results make evident that the reflectivity response provided by the simple circuit model perfectly matches the full-wave results, and that it gives a good physical insight into this complicated response, even in the grating lobe region (diffraction regime). These results also prove the important reduction of computational effort that our approach provides. In the case of Fig. 3(a), around 500 full-wave points were needed to obtain the complete spectrum (red curve), whereas only seven simulations were performed when applying our equivalent-circuit approach (blue curve).

The effect of the angle of incidence can also be modeled and interpreted by the proposed circuit model. The reflectivity of the dipole array previously considered is now shown in Fig. 4 for three different angles of incidence $\left(\theta=0^{\circ}, 40^{\circ}, 80^{\circ}\right)$. In order to characterize our structure up to $60 \mathrm{GHz}$, it is necessary to select the appropriate values of $N_{\mathrm{TE}}$ and $N_{\mathrm{TM}}$ for each angle of incidence. This can be done by looking at Fig. 3(b), where we can see that $N_{\mathrm{TE}}=N_{\mathrm{TM}}=1$ is required to obtain 


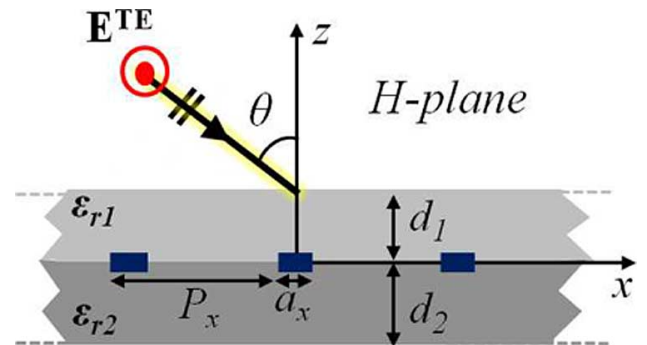

Fig. 5. Scheme of the TE-polarized wave excitation in $H$-plane of the dipole array in Fig. 1 when sandwiched between dielectric slabs.

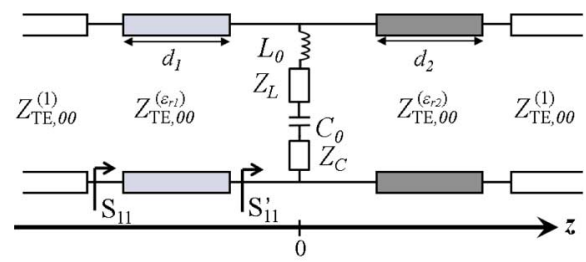

(a)

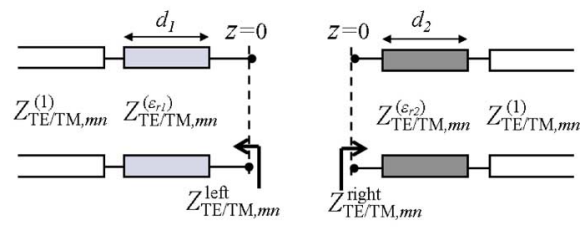

(b)

Fig. 6. (a) Equivalent circuit for the scattering of an obliquely incident plane wave in the periodic array shown in Fig. 5. (b) Transmission line circuits involved in the definition of $Z_{L}$ and $Z_{C}$.

the $\theta=0^{\circ}$ curve in Fig. 4 , whereas $N_{\mathrm{TE}}=2$ and $N_{\mathrm{TM}}=1$ are needed to obtain the curves corresponding to $\theta=40^{\circ}$ and $\theta=80^{\circ}$. Fig. 4(a) shows the extraordinary reflection peaks produced by dipoles of less-than-half wavelength compared to the array periodicity $\left(a_{y}=2 \mathrm{~mm}\right.$, with Fabry-Pérot resonance around $75 \mathrm{GHz}$ ), whereas conventional resonances are shown in Fig. 4(b) for the case of longer dipoles $\left(a_{y}=3.5 \mathrm{~mm}\right.$, with Fabry-Pérot resonance around $40 \mathrm{GHz}$ ). Again, excellent agreement is obtained between full-wave results and the circuit model.

\section{Dipole Array in Stratified Medium}

Once we have discussed the basis of our model for the simpler case of a freestanding dipole-based periodic surface, in this section, we characterize this surface when sandwiched between dielectric slabs (see Fig. 5). The incident wave impinges obliquely on the array in the plane $x z$ ( $H$-plane) with an angle $\theta$. Following the guidelines reported in Section II, the TEN in Fig. 6 is proposed in order to model this structure, where we can observe important changes with respect to the TEN in Fig. 2. First, the propagation of the incident, reflected, and transmitted TE plane wave inside the dielectric slabs is modeled in Fig. 6(a) by the transmission lines of finite length $d_{1}$ and $d_{2}$, which are placed at both sides of the discontinuity. The characteristic impedances associated with these lines are $Z_{\mathrm{TE}, 00}^{\left(\varepsilon_{r 1}\right)}$ and $Z_{\mathrm{TE}, 00}^{\left(\varepsilon_{r 2}\right)}$, respectively, and can be obtained using (8). In order to account for the excitation of higher order harmonics at the discontinuity, it is necessary to introduce the following impedances defined in Fig. 6(b):

$$
\begin{aligned}
& Z_{\mathrm{TE} / \mathrm{TM}, m n}^{\text {left }}(\omega) \\
& =Z_{\mathrm{TE} / \mathrm{TM}, m n}^{\left(\varepsilon_{r 1}\right)} \frac{Z_{\mathrm{TE} / \mathrm{TM}, m n}^{(1)}+j Z_{\mathrm{TE} / \mathrm{TM}, m n}^{\left(\varepsilon_{r 1}\right)} \tan \left(k_{z, m n}^{\left(\varepsilon_{r 1}\right)} d_{1}\right)}{Z_{\mathrm{TE} / \mathrm{TM}, m n}^{\left(\varepsilon_{r 1}\right)}+j Z_{\mathrm{TE} / \mathrm{TM}, m n}^{(1)} \tan \left(k_{z, m n}^{\left(\varepsilon_{r 1}\right)} d_{1}\right)} \\
& Z_{\mathrm{TE} / \mathrm{TM}, m n}^{\text {right }}(\omega) \\
& =Z_{\mathrm{TE} / \mathrm{TM}, m n}^{\left(\varepsilon_{r 2}\right)} \frac{Z_{\mathrm{TE} / \mathrm{TM}, m n}^{(1)}+j Z_{\mathrm{TE} / \mathrm{TM}, m n}^{\left(\varepsilon_{r 2}\right)} \tan \left(k_{z, m n}^{\left(\varepsilon_{r 2}\right)} d_{2}\right)}{Z_{\mathrm{TE} / \mathrm{TM}, m n}^{\left(\varepsilon_{r 2}\right)}+j Z_{\mathrm{TE} / \mathrm{TM}, m n}^{(1)} \tan \left(k_{z, m n}^{\left(\varepsilon_{r 2}\right)} d_{2}\right)} .
\end{aligned}
$$

In similarity to the rationale followed in [13, Ch. 5], the contribution of each TE/TM $m n$th harmonic to the total impedance $Z_{L}(\omega)$ or $Z_{C}(\omega)$ is accounted for by the parallel connection between $Z_{\mathrm{TE} / \mathrm{TM}, m n}^{\text {left }}(\omega)$ and $Z_{\mathrm{TE} / \mathrm{TM}, m n}^{\text {right }}(\omega)$, which can be written as

$$
\begin{aligned}
& Z_{L}(\omega)=\sum_{h=1}^{N_{\mathrm{TE}}} A_{h}^{\mathrm{TE}}\left(\frac{1}{Z_{\mathrm{TE}, h}^{\text {left }}(\omega)}+\frac{1}{Z_{\mathrm{TE}, h}^{\text {right }}(\omega)}\right)^{-1} \\
& Z_{C}(\omega)=\sum_{h=1}^{N_{\mathrm{TM}}} A_{h}^{\mathrm{TM}}\left(\frac{1}{Z_{\mathrm{TM}, h}^{\mathrm{left}}(\omega)}+\frac{1}{Z_{\mathrm{TM}, h}^{\mathrm{right}}(\omega)}\right)^{-1}
\end{aligned}
$$

Looking at the above expressions, the combined influence of both dielectrics in the response of the array can be observed. Specifically, the cutoff of higher order harmonics in the dielectric mediums (onset of the so-called "trapped" surface waves $[13$, Ch. 5]) plays an important and intricate role in the appearance of singularities in $Z_{L}$ and $Z_{C}$ as well as in the subsequent generation of reflectivity resonances and nulls. Here, the capital relevance of setting up the appropriate connections between the different elements in order to make the equivalent network physically meaningful should be emphasized. Otherwise, the TEN would be no more than a numerical fitting procedure valid in a certain frequency region. From the proposed TEN, the following identity can be written:

$$
\begin{aligned}
j \omega L_{0} & +Z_{L}(\omega)+\frac{1}{j \omega C_{0}}+Z_{C}(\omega) \\
& =\left(\frac{1-S_{11}^{\prime}(\omega)}{\left[1+S_{11}^{\prime}(\omega)\right] Z_{\mathrm{TE}, 00}^{(1)}(\omega)}-\frac{1}{Z_{\mathrm{TE}, 00}^{\mathrm{in}}(\omega)}\right)^{-1}
\end{aligned}
$$

where $S_{11}^{\prime}$ is the reflection coefficient at the reference plane $z=0$, as shown in Fig. 6(a). This coefficient can readily be expressed in terms of the reflection coefficient $S_{11}$ at $z=-d_{1}$ [53], which is the coefficient that can directly be obtained from full-wave simulations [34]. Thus, the parameters $L_{0}, C_{0}$, and $A_{h}^{\mathrm{TE} / \mathrm{TM}}$ can be obtained after solving the system of equations resulting from particularizing (16) at a few values of frequency. Similar to the case of freestanding dipoles, as many full-wave data points as the number of unknowns are needed. The choice of the evaluated frequency values basically follows the same general rules reported in the previous section. However, in the present case, we propose to take the high-frequency points near 
and below the onset of the higher order harmonics in the denser dielectric medium.

The cutoff frequency of higher order harmonics is lower inside a dielectric medium than in free space. Thus, the frequency range satisfying $f_{c, m n}^{\left(\varepsilon_{r}\right)}<f<f_{c, m n}^{(1)}$ corresponds to the existence of harmonics that are propagative in the dielectric slab but that are still evanescent in free space. Hence, in this range, trapped surface waves appear below the grating lobe regime [13]. [The grating lobe regime is still determined by the frequency at which the first higher order harmonic becomes propagative in free space; $60 \mathrm{GHz}$ in the present case for normal incidence, as shown in Fig. 3(b)]. As a consequence, the total amount of higher order harmonics that should be considered in the TEN increases with respect to the freestanding case. Since (14) and (15) show that the dielectric slabs contribute as a whole [13], the proposed number of higher order harmonics $\left(N_{\mathrm{TE}}\right.$ and $N_{\text {TM }}$ ) is now one plus the number of launched trapped surface waves in the denser slab. This rule should be modified to two plus the number of launched trapped surface waves in the denser slab when the thickness of the slab is similar to or larger than the slab wavelength.

\section{A. Identical Slabs}

The generation of trapped surface waves due to the presence of the dielectric slabs considerably increases the complexity of the situation [13] with respect to the freestanding case. Thus, the case of identical slabs is first considered in order to reach gradually the most complex case. This scenario corresponds to $d_{1}=d_{2}=d$ and $\varepsilon_{r 1}=\varepsilon_{r 2}=\varepsilon_{r}$ in Figs. 5 and 6 . Note that the input impedances defined in (12) and (13), which are associated with the excitation of higher order harmonics at each side of the discontinuity, are equal in the case of identical dielectric slabs. Therefore, the following input impedance can now be defined:

$$
\begin{aligned}
& Z_{\mathrm{TE} / \mathrm{TM}, m n}^{\mathrm{in}}(\omega) \\
& =\frac{1}{2} Z_{\mathrm{TE} / \mathrm{TM}, m n}^{\mathrm{right}}(\omega)=\frac{1}{2} Z_{\mathrm{TE} / \mathrm{TM}, m n}^{\text {left }}(\omega) \\
& =\frac{1}{2} Z_{\mathrm{TE} / \mathrm{TM}, m n}^{\left(\varepsilon_{r}\right)} \frac{Z_{\mathrm{TE} / \mathrm{TM}, m n}^{(1)}+j Z_{\mathrm{TE} / \mathrm{TM}, m n}^{\left(\varepsilon_{r}\right)} \tan \left(k_{z, m n}^{\left(\varepsilon_{r}\right)} d\right)}{Z_{\mathrm{TE} / \mathrm{TM}, m n}^{\left(\varepsilon_{r}\right)}+j Z_{\mathrm{TE} / \mathrm{TM}, m n}^{(1)} \tan \left(k_{z, m n}^{\left(\varepsilon_{r}\right)} d\right)}
\end{aligned}
$$

which makes the analogy between this situation and the one presented in Section II clearer. Fig. 7 shows the cutoff frequency chart versus the angle of incidence in a dielectric medium with $\varepsilon_{r}=3$, and the same array periodicity as in previous examples $\left(P_{x}=P_{y}=5 \mathrm{~mm}\right)$. For normal incidence, the onset of the trapped surface waves occurs at 35 and $49 \mathrm{GHz}$, well below the starting frequency of the grating lobe regime $(60 \mathrm{GHz})$. As a result, two singularities appear at approximately 47.3 and $62.5 \mathrm{GHz}$ in $Z_{\mathrm{TE} / \mathrm{TM}, m n}^{\mathrm{in}}(\omega)$, where the denominator of (17) is zero (more specifically, at 47.3 and $62.5 \mathrm{GHz}$ for the $\mathrm{TE}_{10}$ and $\mathrm{TE}_{11}$ harmonics, respectively). At these frequencies, $Z_{L}$ diverges, causing an open circuit in the branch of series elements that account for the effect of the dipoles in Fig. 6(a). Since this open circuit makes this branch irrelevant, the complete structure should behave as if the dipoles were not present.

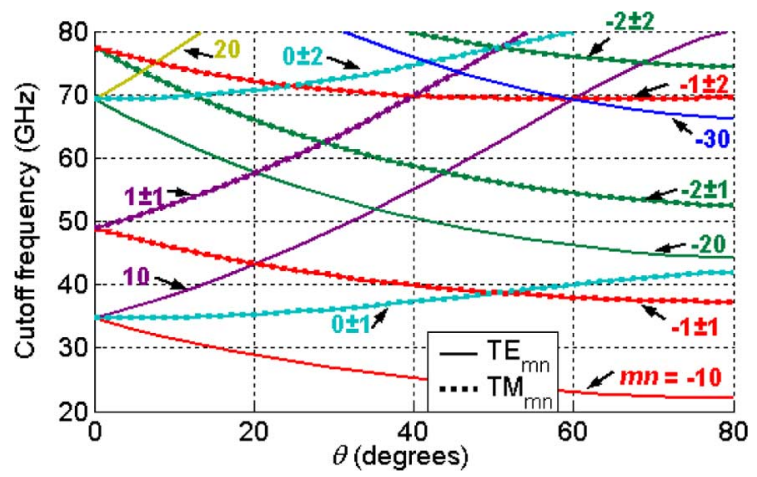

Fig. 7. Cutoff frequency of higher order harmonics for $\varepsilon_{r}=3$.

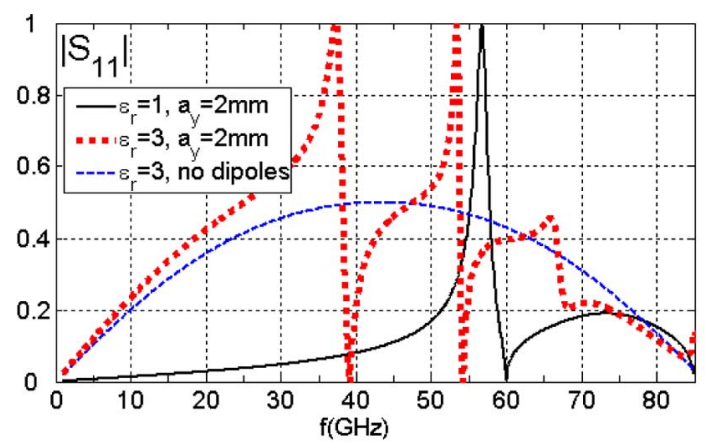

(a)

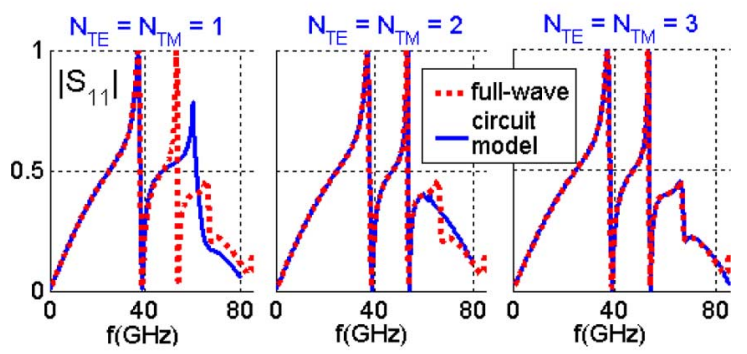

(b)

Fig. 8. (a) Magnitude of the full-wave reflection coefficient at normal incidence for the structure in Fig. 5 with $\varepsilon_{r 1}=\varepsilon_{r 2}=\varepsilon_{r}, d_{1}=d_{2}=0.5 \mathrm{~mm}$, $P_{x}=P_{y}=5 \mathrm{~mm}$, and $a_{x}=0.5 \mathrm{~mm}$. (b) For the case of the red dotted line in Fig. 8(a), comparison between full-wave and circuit model results (configurations: $N_{\mathrm{TE}}=N_{\mathrm{TM}}=1, N_{\mathrm{TE}}=N_{\mathrm{TM}}=2$, and $N_{\mathrm{TE}}=N_{\mathrm{TM}}=3$, respectively).

The above fact is corroborated in Fig. 8(a) at 47.3 and $62.5 \mathrm{GHz}$ where the dashed blue curve (which shows the behavior of the structure without dipoles) crosses with the dotted red curve (with dipoles). Fig. 8(a) shows two reflectivity resonances below the grating lobe regime for the dielectric sandwiched array with dipole length $a_{y}=2 \mathrm{~mm}$. These two resonances are of Fano type, in similarity with the freestanding case. However, in this latter case, the solid back line in Fig. 8(a) shows that only one Fano resonance can occur below the grating lobe regime. Once within the grating lobe regime, leakage to secondary grating lobes prevent the appearance of additional total reflection peaks [see Fig. 8(a) above $60 \mathrm{GHz}$ ]. The reflection nulls in the dashed red line in Fig. 8(a) do not appear at the cutoff frequencies of the trapped surface waves (35 and $49 \mathrm{GHz}$ ), as happened in the freestanding case. In the presence of dielectrics, the impedance matching condition 


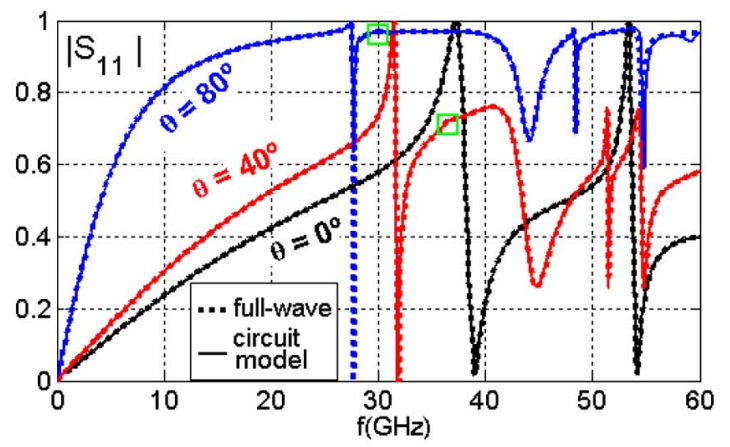

(a)
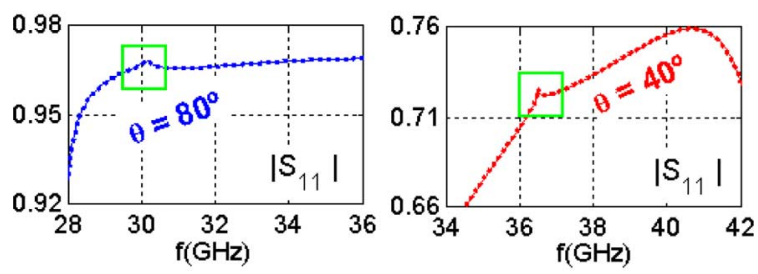

(b)

Fig. 9. Magnitude of the reflection coefficient for the structure in Fig. 5 with $d_{1}=d_{2}=0.5 \mathrm{~mm}, \varepsilon_{r 1}=\varepsilon_{r 2}=\varepsilon_{r}=3$ and $a_{y}=2 \mathrm{~mm}$ (unit cell: $P_{x}=P_{y}=5 \mathrm{~mm}$ and $a_{x}=0.5 \mathrm{~mm}$ ). (a) Different angles of incidence. (b) Details of the appearance of grating lobes.

[46] responsible for the reflection nulls requires that the input impedance at $z=-d$ equals that of the incident transmission line. Although this condition is not easily expressible in closed form, it can be observed that it does not only depend on the unit-cell dimensions but also on the characteristics of the dielectric slab as well as on the dipole geometry. (This latter dependence comes through the excitation parameters $A_{h}^{\mathrm{TE} / \mathrm{TM}}$ appearing in $Z_{L}$ and $Z_{C}$.)

In order to investigate the role played by the dielectric-slab higher order harmonics on the behavior of the array reflectivity, Fig. 8(b) shows the results obtained using different numbers of harmonics. If only the first TE and TM higher order harmonics $\left(\mathrm{TE}_{10}\right.$ and $\left.\mathrm{TM}_{01}\right)$ are considered, the subplot $N_{\mathrm{TE}}=N_{\mathrm{TM}}=1$ in Fig. 8(b) shows that the reflectivity response is accurately modeled up to the frequency of the first resonance and subsequent null $(f \lesssim 39 \mathrm{GHz})$. The addition of extra harmonics is needed to retrieve the following nulls and associated maxima. The addition of the second TE and TM harmonic $\left(\mathrm{TE}_{11}\right.$ and $\mathrm{TM}_{11}$ ) implies that the second trapped surface wave inside the dielectric is now considered. The effect of these additional harmonics is depicted in the subplot $N_{\mathrm{TE}}=N_{\mathrm{TM}}=2$, which shows now a good agreement for the two Fano resonances. Finally, the subplot $N_{\mathrm{TE}}=N_{\mathrm{TM}}=3$ in Fig. 8(b) shows the effect of the addition of the third TE and TM harmonic ( $\mathrm{TE}_{20}$ and $\mathrm{TM}_{02}$ ). It can be seen that this addition helps to accurately reproduce the reflectivity local maximum at $66 \mathrm{GHz}$, which fails to be total reflection within the grating lobe regime.

In Fig. 9(a), the effect of increasing the angle of incidence is shown. At normal incidence, we can observe two extraordinary total reflection peaks but only one of these peaks remains for the other incidence angles here considered (which do show several nontotal reflection peaks). As can be seen in the cutoff chart for the dielectric medium with $\varepsilon_{r}=3$ (Fig. 7), the cutoff of the first higher order TE harmonic decreases from 35 to $25 \mathrm{GHz}$ as $\theta$ increases from $0^{\circ}$ to $40^{\circ}$. Related to this fact, the lowest resonance frequency also decreases in Fig. 9(a) from $\sim 38 \mathrm{GHz}$ at normal incidence to $\sim 32 \mathrm{GHz}$ at $\theta=40^{\circ}$. However, it should also be observed that the grating lobe frequency [shown in the free-space cutoff chart in Fig. 3(b)] also decreases from $60 \mathrm{GHz}$ at $\theta=0^{\circ}$ to $36.52 \mathrm{GHz}$ at $\theta=40^{\circ}$. Consequently, no additional total-reflection peaks are observed in Fig. 9(a) for $\theta=40^{\circ}$. At the onset of the grating lobes, an inflection in the reflectivity response is observed. This detail is amplified in the subplot $\theta=40^{\circ}$ of Fig. 9(b). The reflectivity response for $\theta=80^{\circ}$ bears some similarities to that observed for $\theta=40^{\circ}$. Thus, the $\theta=80^{\circ}$ curve in Fig. 9(a) shows a single extraordinary reflection peak that is shifted to lower frequencies $(\sim 27.5 \mathrm{GHz})$, followed by a closer grating lobe onset now located at $30 \mathrm{GHz}$ [see Fig. 3(b)]. A similar inflection point is also observed at $30 \mathrm{GHz}$ in the subplot $\theta=80^{\circ}$ of Fig. 9(b). It should be highlighted that the circuit-model approach perfectly matches the complicated spectrum given by the full-wave method, including all the fine details of very sharp resonances, inflection points, partial maxima and minima, etc.

\section{B. Different Slabs}

Once the symmetric scenario has been analyzed, we can now use the experience gained in this case for the analysis of a dipole array sandwiched between arbitrary dielectric slabs. In this situation, the cutoff of higher order harmonics in both dielectric slabs is again related to the appearance of singularities in $Z_{L}$ and $Z_{C}$. Therefore, the same discussion about the launch of trapped surface waves in the symmetric case can now be applied. One particular scenario of great practical interest is found when the metallic arrays of dipoles are printed on a dielectric slab [12], [14], [30], [34]-[36], [38], [39], [49]. In order to validate our TEN in this particular situation, the same unit cell previously studied and described in the caption of Fig. 1 is now modeled when printed on a thin supporting substrate. For this purpose, it is assumed that the upper dielectric in Fig. 5 is free space $\left(d_{1}=0\right)$ whereas the lower supporting substrate has $d_{2}=0.5 \mathrm{~mm}, \varepsilon_{r 2}=3$. As an example study of this structure, Fig. 10 shows the reflectivity under plane wave oblique incidence $\left(\theta=40^{\circ}\right)$ for both electrically small and long dipoles ( $a_{y}=2 \mathrm{~mm}$ and $a_{y}=3.5 \mathrm{~mm}$, respectively). Apart from the excellent agreement again observed between our equivalent circuit approach and the full-wave simulations, some physical explanations for these results are once more given by the circuit model. In Fig. 9(a), we can observe that only one total reflection peak $\left(\left|S_{11}\right|=1\right)$ is found in both curves below the grating lobe regime. This regime is reached at $36.53 \mathrm{GHz}$ in both cases, which is clearly manifested as an inflection in the $\left|S_{11}\right|$ curves. However, instead of the reflectivity nulls below the grating lobe regime in Fig. 9(a), only local minima are found in Fig. 10. In fact, total transmission $\left(\left|S_{11}\right|=0\right)$ is not expected to occur easily when the dielectric slabs are not identical (the conditions to have impedance matching at $z=-d_{1}$ are now much harder to obtain owing to the parallel connection of the "left" and "right" impedances). The same cutoff frequency chart of Fig. 7 is now employed to set the values of the significant TE and TM harmonics. In particular, the results plotted in Fig. 10 


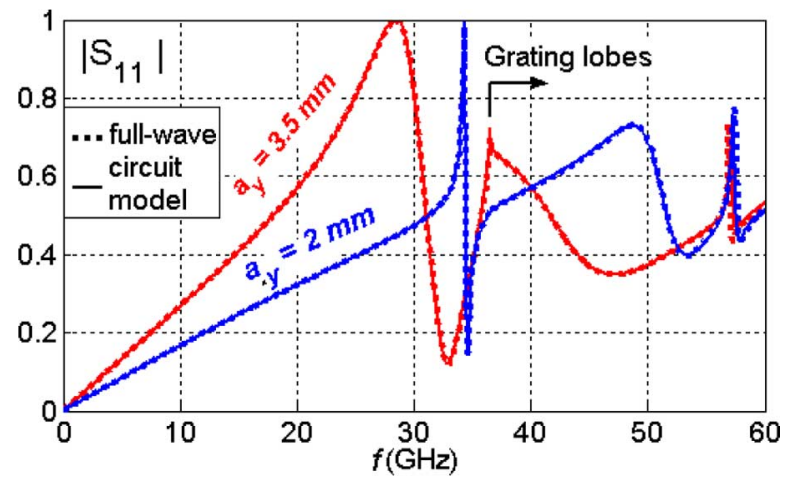

Fig. 10. Magnitude of the reflection coefficient under $40^{\circ}$ incidence for the structure of Fig. 5 with $\varepsilon_{r 1}=1, \varepsilon_{r^{2}}=3, d_{1}=0 \mathrm{~mm}, d_{2}=0.5 \mathrm{~mm}, P_{x}=$ $P_{y}=5 \mathrm{~mm}$, and $a_{x}=0.5 \mathrm{~mm}$.

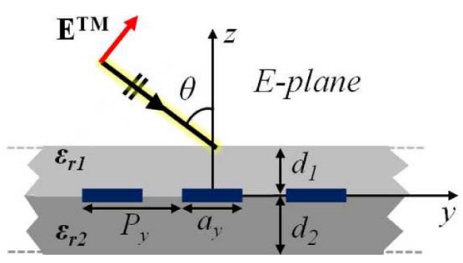

(a)

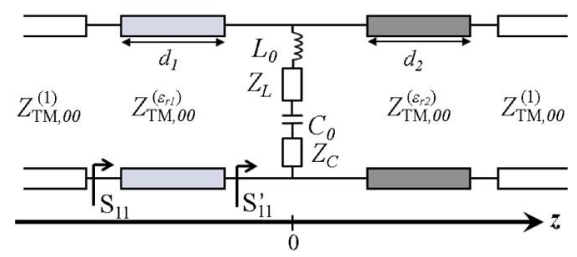

(b)

Fig. 11. (a) TM-polarized wave excitation in $E$-plane of a dipole array sandwiched between dielectric slabs. (b) Proposed circuit model.

have been obtained with $N_{\mathrm{TE}}=7$ and $N_{\mathrm{TM}}=4$, which are equal to one plus the number of TE and TM harmonics above cutoff in the dielectric below $60 \mathrm{GHz}$, respectively.

\section{Dipole ARray Under TM InCIDENCE}

The methodology previously used in the study of the TE incidence case can equally be applied when the exciting plane wave is TM polarized or it impinges in other planes. In practice, partially reflecting sheet arrays are normally illuminated by a single central feed [1], [12], [14], [30], [34]-[36], [38], [39], [48], with the feeding element oriented so that the excited polarized electric field has a strong interaction with the dipoles. Therefore, the characterization of the periodic array under TM polarization becomes relevant when the scan plane of incidence is $y z$ ( $E$-plane). An equivalent network can also be proposed for TE polarization in $E$-plane or TM in $H$-plane, although these cases are less relevant from a practical point of view. Thus, in this section, the incidence of a TM-polarized plane wave in the $E$-plane of a dipole array [illustrated in Fig. 11(a)] is modeled by the circuit model shown in Fig. 11(b). The only difference between this TEN and the one in Fig. 6(a) is in the transmission lines at both sides of the discontinuity. The transmission lines now model the propagation of the incident, reflected, and

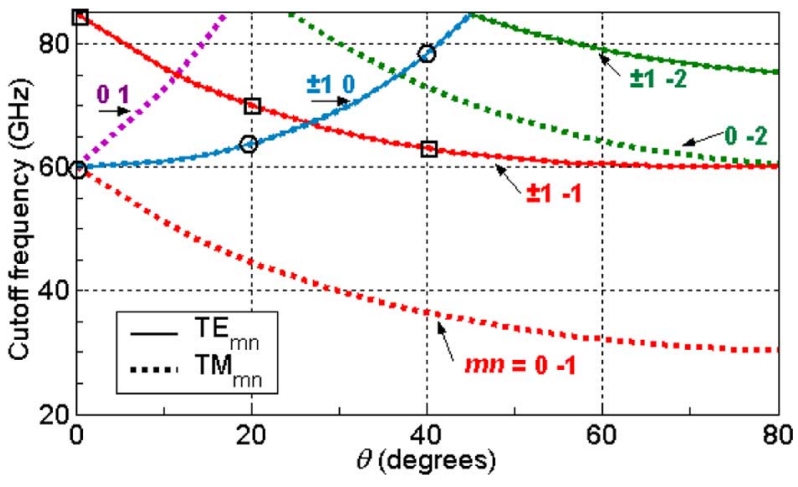

Fig. 12. Higher order harmonic cutoff frequency in free space (unit cell with periodicities $P_{x}=P_{y}=5 \mathrm{~mm}$ ).

transmitted $\mathrm{TM}_{00}$ harmonic. As a consequence, the same procedure explained in Section III can here be employed. The impedances $Z_{L}$ and $Z_{C}$ in Fig. 11(b) are again defined by (14) and (15). Equation (16) can also be solved for the unknown values of $L_{0}, C_{0}$, and $A_{m n}^{\mathrm{TE} / \mathrm{TM}}$ considering that $S_{11}^{\prime}$ is the reflection coefficient plotted in Fig. 11(b). Due to the change in the plane of incidence, the excited harmonics now propagate along the $x$ - and $y$-axis with the following wavenumbers $(m$, $n=0, \pm 1, \pm 2, \ldots)$ :

$$
\begin{aligned}
& k_{x, m}=\frac{2 m \pi}{P_{x}} \\
& k_{y, n}=k_{0} \sin \theta+\frac{2 n \pi}{P_{y}} .
\end{aligned}
$$

The cutoff frequencies associated with higher order harmonics can be computed as

$$
\begin{aligned}
f_{c, m n}^{\left(\varepsilon_{r}\right)} & \frac{1}{\varepsilon_{r}-\sin ^{2} \theta} \\
& \times\left\{\frac{n c}{P_{y}} \sin \theta+\sqrt{\varepsilon_{r}\left[\left(\frac{n c}{P_{y}}\right)^{2}+\left(\frac{m c}{P_{x}}\right)^{2}\right]-\left(\frac{m c}{P_{x}} \sin \theta\right)^{2}}\right\} .
\end{aligned}
$$

The periodic problem under consideration can be reduced to a single unit-cell problem with magnetic walls parallel to the $y z$-plane and Floquet walls (periodic boundary conditions) parallel to the $x z$-plane. Therefore, $\mathrm{TE}_{0 n}$ harmonics do not satisfy the boundary conditions, and are not excited by the TM polarized impinging wave. This fact is illustrated in Fig. 12, which shows the higher order cutoff frequencies computed for free space in a unit cell with periodicities $P_{x}=P_{y}=5 \mathrm{~mm}$. In contrast to Fig. 3(b), the first grating lobe is now produced by the harmonic $\mathrm{TM}_{0-1}$, instead of $\mathrm{TE}_{-10}$.

Our first study for TM incidence is the freestanding case shown in Fig. 13(a), where the reflectivity of a dipole array with dimensions $P_{x}=P_{y}=5 \mathrm{~mm}, a_{x}=0.5 \mathrm{~mm}$, and $a_{y}=2 \mathrm{~mm}$ is shown. Normal TE incidence in $H$-plane and TM incidence in $E$-plane are equal and, therefore, the $\theta=0^{\circ}$ curves in Figs. 4(a) and 13(a) are identical. As was explained in Section III, for 


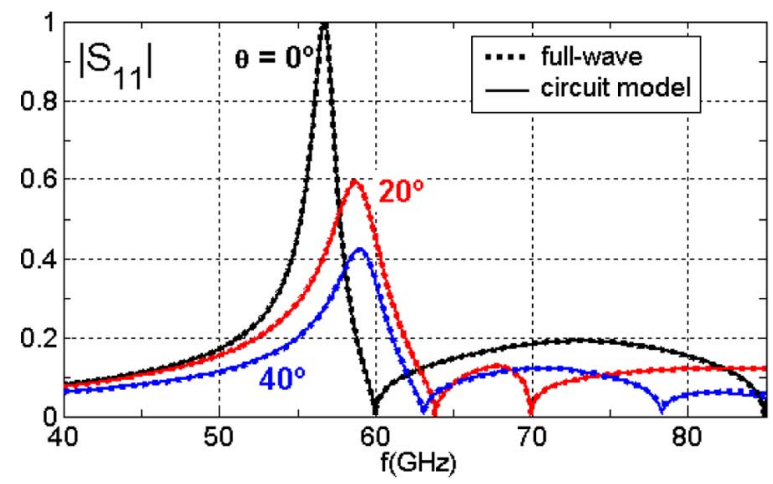

(a)

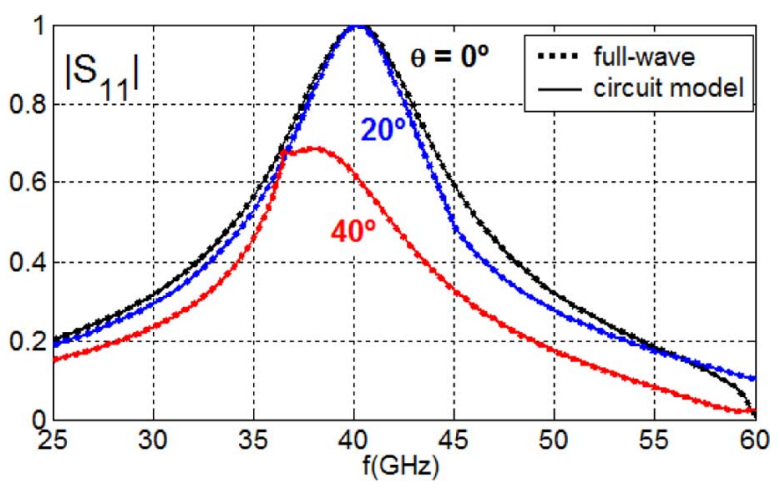

(b)

Fig. 13. Magnitude of the reflection coefficient for the structure described in Fig. 1 under oblique $E$-plane TM incidence. (a) Dipole length $a_{y}=2 \mathrm{~mm}$. (b) $a_{y}=3.5 \mathrm{~mm}$.

normal incidence, an extraordinary transmission peak appears at $56.7 \mathrm{GHz}$, before the onset of the first TE harmonic. For each angle of incidence, Wood's anomalies (reflection nulls) found in Fig. 13(a) exactly appear at the onset of TE harmonics, analytically predicted in Fig. 12. Specifically, the reflectivity null associated to the onset of the harmonic $\mathrm{TE}_{10}$ increases from $60 \mathrm{GHz}$ at $0^{\circ}$ to $63.9 \mathrm{GHz}$ at $20^{\circ}$ and $78.4 \mathrm{GHz}$ at $40^{\circ}$ (as marked with black circles in Fig. 12). In contrast, the onset of the harmonic $\mathrm{TE}_{1-1}$ decreases from $85 \mathrm{GHz}$ at $0^{\circ}$ to $70 \mathrm{GHz}$ at $20^{\circ}$, and $63.1 \mathrm{GHz}$ at $40^{\circ}$ (marked with black squares in Fig. 12). It should be recalled that the beginning of the grating lobes regime is now set by a TM harmonic (see $\mathrm{TM}_{0-1}$ curve in Fig. 12), which does not diverge at its cutoff, and therefore, it does not produce a singularity in the reflection coefficient. The consequence of this is the suppression of the extraordinary total reflection for oblique incidence, as shown in Fig. 13(a). The divergence of the first TE harmonic occurs in the grating lobes regime, where (as was explained in Section II), no total reflection can be produced. Yet, a peak of maximum reflectivity can still be found before the onset of the first TE harmonic, whose level decreases as $\theta$ increases. Fig. 13(b) also shows the reflectivity response of the previous unit cell when $a_{y}=$ $3.5 \mathrm{~mm}$. The $\theta=0^{\circ}$ curve again coincides with the one in Fig. 4(b), and as was explained in Section II, conventional total reflection appears at $40 \mathrm{GHz}$. This resonance may also be suppressed when the onset of the $\mathrm{TM}_{0-1}$ harmonic is lower that the quasi-static resonance of the dipole, as is illustrated

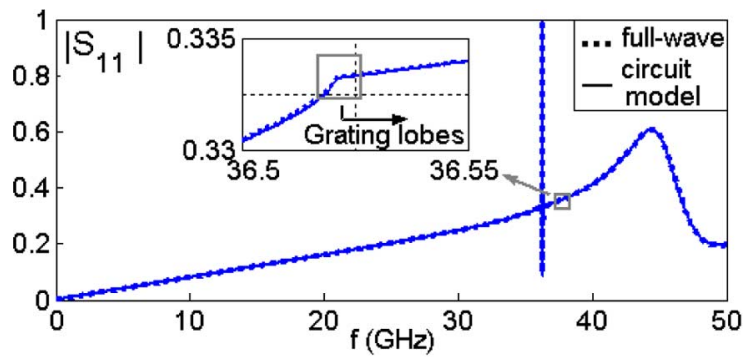

(a)

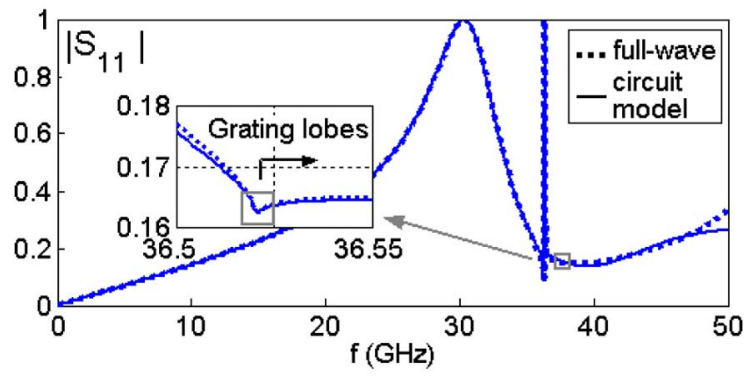

(b)

Fig. 14. Magnitude of the reflection coefficient under $\theta=40^{\circ} \mathrm{TM}$ incidence on $E$-plane for the structure of Fig. 11 with $P_{x}=P_{y}=5 \mathrm{~mm}, a_{x}=0.5 \mathrm{~mm}$, $\varepsilon_{r 1}=1, \varepsilon_{r 2}=3, d_{1}=0 \mathrm{~mm}$, and $d_{2}=0.5 \mathrm{~mm}$. (a) Dipole length $a_{y}=$ $2 \mathrm{~mm}$; (b) $a_{y}=3.5 \mathrm{~mm}$.

by curve $\theta=40^{\circ}$ in Fig. 13(b) $\left(\mathrm{TM}_{0-1}\right.$ cutoff frequency is $36.53 \mathrm{GHz}$, lower than $40 \mathrm{GHz}$ ). In this case, the onset of the first TE harmonic is not directly responsible for the appearance of the conventional reflection peak. Therefore, in contrast to what happens in Fig. 13(a), the resonance does not disappear for every incidence greater than zero. For example, Fig. 13(b) shows that total reflection still occurs when $\theta=20^{\circ}$, due to the fact that the onset of the $\mathrm{TM}_{0-1}$ harmonic is above $40 \mathrm{GHz}$ (45 GHz as shown in Fig. 12). In Fig. 13(b), an inflection in the reflectivity response can be observed at the onset of the grating lobes.

The practical design studied in Section III of a dipole array printed on a thin dielectric substrate can again be modeled under TM incidence in E-plane using the TEN proposed in Fig. 11(b). Fig. 14 shows the reflection coefficient presented by this printed structure under oblique incidence $\left(\theta=40^{\circ}\right)$ for electrically small [Fig. 14(a)] and long dipoles [Fig. 14(b)]. The onset of the grating lobe regime causes again an inflection at $36.53 \mathrm{GHz}$, which has been zoomed in on each figure. The existence of a trapped wave inside the dielectric slab gives rise to a short circuit in the branch of series impedances in Fig. 11(b) at a frequency close to the onset of the grating harmonic $\mathrm{TM}_{0-1}$. After this reflection peak, there quickly appears a reflection dip that causes an extraordinary (Fano) resonance before $36.53 \mathrm{GHz}$ (see Fig. 14). In addition, the conventional resonance peak has not been suppressed in Fig. 14(b) since it now appears at a frequency below the onset of the first grating lobe. The circuit-model results shown in Fig. 14 have been obtained using $N_{\mathrm{TE}}=3$ and $N_{\mathrm{TM}}=4$ in (14) and (15) according to the general rules previously reported.

Before finishing our study we would like to highlight that our equivalent-circuit model can also be used to characterize 


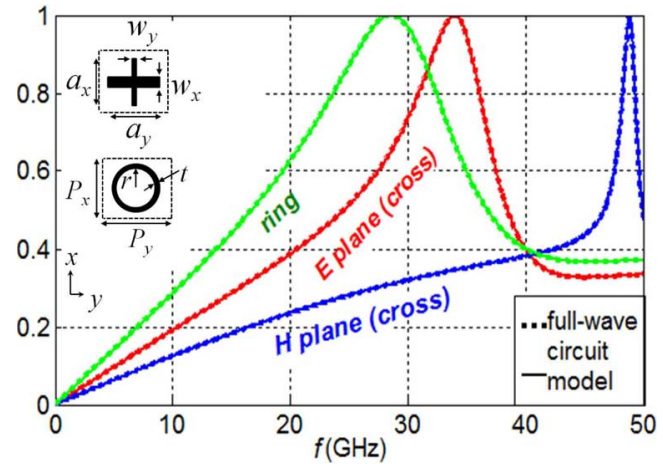

Fig. 15. Magnitude of the reflection coefficient under TM normal incidence for periodic arrangements $\left(P_{x}=P_{y}=5 \mathrm{~mm}\right)$ of circular rings $(r=3 \mathrm{~mm}$, $t=0.5 \mathrm{~mm})$ and cross dipoles $\left(a_{x}=2 \mathrm{~mm}, a_{y}=3.5 \mathrm{~mm}\right.$, and $w_{x}=w_{y}=$ $0.5 \mathrm{~mm}$ ). The arrays are printed on a substrate of $\varepsilon_{r}=2, d=1 \mathrm{~mm}$.

structures with more complicated geometries of the scatterers, with the condition that their current profile does not change significantly with frequency. This includes many cases of practical interest, such as those recently reported in [56]-[58]. As representative examples, next we study two arrays of different printed elements (i.e., a cross dipole and a symmetric ring) under TM normal incidence in Fig. 15. In the cross-dipole case, the reflectivity response has been studied when the exciting TM plane wave impinges either in the $H$-plane or the $E$-plane (i.e., the electric field is polarized along the $x$ - or $y$-axis, respectively). The cross-dipole scatterers are sensitive to both polarizations since they have long metallization along both the $x$ and $y$-directions. As can be inferred from the previous analysis with simple metallic dipoles, the total reflection peaks found at $48.5 \mathrm{GHz}$ in the $H$-plane and $34 \mathrm{GHz}$ in the $E$-plane are mainly related to the dimensions $a_{x}$ and $a_{y}$, respectively. When dealing with a symmetric ring structure, the same results are obtained in both planes for normal incidence. The total reflection peak at $28.6 \mathrm{GHz}$ in Fig. 15 appears when the wavelength is approximately the perimeter of the ring [55]. The good agreement with full-wave simulations proves the versatility of our approach.

\section{CONCLUSION}

A systematic approach to the circuit modeling of the reflection/transmission features of 2-D arrays of printed/free standing conducting dipoles has been described in this paper. This approach is based on the physical modal decomposition of the electromagnetic fields around the scatterers (printed dipoles in our case). The contribution of the infinite number of very high-order modes scattered by the printed surface can be represented by a simple inductor and a simple capacitor. Only TE and TM modes with cutoff frequencies in the region of interest must be treated explicitly. After setting up the appropriate connection characteristics of the proposed equivalent network, the specific contribution of these few modes can be extracted by solving a small linear system of equations whose coefficients come from a few full-wave numerical simulations carried out at certain specific frequency points. The accuracy of the circuit model predictions has been demonstrated for two different polarizations and a wide range of frequencies and incidence angles. The availability of a circuit model not only reduces the overall computational effort but, even more importantly, it also provides a theoretical frame for understanding the qualitative performance of the patterned surface and to design devices based on such structures. In particular, the extraordinary reflection behavior that has given place to various and controversial theories in the past is very simply accounted for by our model.

\section{REFERENCES}

[1] G. V. Trentini, "Partially reflecting sheet arrays," IRE Trans. Antennas Propag., vol. AP-4, no. 4, pp. 666-671, Oct. 1956.

[2] A. A. Oliner, "Equivalent circuit for small symmetrical longitudinal apertures and obstacles," IRE Trans. Microw. Theory Tech., vol. MTT-8, no. 1, pp. 72-80, Jan. 1960.

[3] I. Palocz and A. A. Oliner, "Equivalent network of a multimode planar grating," IEEE Trans. Microw. Theory Tech., vol. MTT-18, no. 5, pp. 244-252, May 1970.

[4] C.-C. Chen, "Diffraction of electromagnetic waves by a conducting screen perforated periodically with circular holes," IEEE Trans. Microw. Theory Tech., vol. MTT-19, no. 5, pp. 475-481, May 1971.

[5] C.-C. Chen, "Transmission of microwave through perforated flat plates of finite thickness," IEEE Trans. Microw. Theory Tech., vol. MTT-21, no. 1, pp. 1-6, Jan. 1973.

[6] M. Guglielmi and A. A. Oliner, "Multimode network description of a planar periodic metal-strip grating at a dielectric interface-Part I: Rigorous network formulations," IEEE Trans. Microw. Theory Tech., vol. 37, no. 3, pp. 535-541, Mar. 1989.

[7] M. Guglielmi and A. A. Oliner, "Multimode network description of a planar periodic metal-strip grating at a dielectric interface-Part II: Small-aperture and small-obstacle solutions," IEEE Trans. Microw. Theory Tech., vol. 37, no. 3, pp. 542-552, Mar. 1989.

[8] R. Ulrich, "Far-infrared properties of metallic mesh and its complementary structure," Infrared Phys., vol. 7, pp. 37-55, 1967.

[9] R. Ulrich, "Effective low-pass filters for far infrared frequencies," Infrared Phys., vol. 7, pp. 65-74, 1967.

[10] C. T. Cunningham, "Resonant grids and their use in the construction of submillimeter filters," Infrared Phys., vol. 23, no. 4, pp. 207-215, 1983.

[11] P. G. J. Irwin, P. A. R. Ade, S. B. Calcutt, F. W. Taylor, J. S. Seeley, R. Hunneman, and L. Walton, "Investigation of dielectric spaced resonant mesh filter designs for PMIRR," Infrared Phys., vol. 34, no. 6, pp. 549-563, 1993.

[12] J. C. Vardaxoglou, Frequency Selective Surfaces Analysis and Design. Taunton, U.K.: Research Studies, 1997.

[13] B. Munk, Frequency Selective Surfaces: Theory and Design. New York: Wiley, 2000.

[14] A. P. Feresidis and J. C. Vardaxoglou, "High gain planar antenna using optimised partially reflective surfaces," IEE Proc. Microw. Antennas Propag., vol. 148, no. 6, pp. 345-350, Dec. 2001.

[15] O. Luukkonen, C. Simovski, G. Granet, G. Goussetis, D. Lioubtchenko, A. V. Räisänen, and S. A. Tretyakov, "Simple and accurate analytical model of planar grids and high-impedance surfaces comprising metal strips or patches," IEEE Trans. Antennas Propag., vol. 56, no. 6, pp. 1624-1632, Jun. 2008.

[16] F. Bayatpur and K. Sarabandi, "Single-layer higher-order miniaturized-element frequency-selective surfaces," IEEE Trans. Microw. Theory Tech., vol. 56, no. 4, pp. 774-781, Apr. 2008.

[17] F. Bayatpur and K. Sarabandi, "Multipole spatial filters using metamaterial-based miniaturized-element frequency-selective surfaces," IEEE Trans. Microw. Theory Tech., vol. 56, no. 12, pp. 2742-2747, Dec. 2008.

[18] M. Al-Joumayly and N. Behdad, "A new technique for design of low-profile, second-order, bandpass frequency selective surfaces," IEEE Trans. Antennas Propag., vol. 57, no. 2, pp. 452-459, Feb. 2009.

[19] N. Behdad, M. Al-Joumayly, and M. Salehi, "A low-profile third-order bandpass frequency selective surface," IEEE Trans. Antennas Propag., vol. 57, no. 2, pp. 460-466, Feb. 2009. 
[20] R. W. Wood, "On a remarkable case of uneven distribution of light in a diffraction grating spectrum," Philos. Mag., vol. 4, pp. 396-402, 1902.

[21] L. Rayleigh, "Note on the remarkable case of diffraction spectra described by Prof. Wood," Philos. Mag., vol. 14, pp. 60-65, 1907.

[22] T. W. Ebbesen, H. J. Lezec, H. F. Ghaemi, T. Thio, and P. A. Wolff, "Extraordinary optical transmission through sub-wavelength hole arrays," Nature, vol. 391, pp. 667-669, Feb. 1998.

[23] C. Genet and T. W. Ebbesen, "Light in tiny holes," Nature, vol. 445, pp. 39-46, Jan. 2007.

[24] F. J. García de Abajo, "Colloquium: Light scattering by particle and hole arrays," Rev. Mod. Phys., vol. 79, pp. 1267-1290, Oct.-Dec. 2007.

[25] K. Y. Bliokh, Y. P. Bliokh, V. Freilikher, S. Savel'ev, and F. Nori, "Colloquium: Unusual resonators: Plasmonics, metamaterials, and random media," Rev. Mod. Phys., vol. 80, pp. 1201-1213, Oct.-Dec. 2008.

[26] F. J. García-Vidal, L. Martín-Moreno, T. W. Ebbesen, and L. Kuipers, "Light passing through subwavelength apertures," Rev. Mod. Phys., vol. 82, pp. 729-787, Jan.-Mar. 2010

[27] R. Gordon, A. G. Brolo, D. Sinton, and K. L. Kavanagh, "Resonant optical transmission through hole-arrays in metal films: Physics and applications," Laser Photon. Rev., vol. 4, no. 2, pp. 311-335, 2010

[28] M. Beruete, M. Sorolla, I. Campillo, J. S. Dolado, L. Martín-Moreno, J. Bravo-Abad, and F. J. García-Vidal, "Enhanced millimeter-wave transmission through subwavelength hole arrays," Opt. Lett., vol. 29, no. 21, pp. 2500-2502, Nov. 2004

[29] D. Sievenpiper, L. Zhang, F. J. Broas, N. G. Alexopulos, and E. Yablonovitch, "High-impedance electromagnetic surfaces with a forbidden frequency band," IEEE Trans. Microw. Theory Tech., vol. 47, no. 11, pp. 2059-2074, Nov. 1999.

[30] S. Maci, M. Caiazzo, A. Cucini, and M. Casaletti, "A pole-zero matching method for EBG surfaces composed of a dipole FSS printed on a grounded dielectric slab," IEEE Trans. Antennas Propag., vol. 53, no. 1, pp. 70-81, Jan. 2005.

[31] F. C. Seman, R. Cahill, V. F. Fusco, and G. Goussetis, "Design of a Salisbury screen absorber using frequency selective surfaces to improve bandwidth and angular stability performance," IET Microw. Antennas Propag., vol. 5, no. 2, pp. 149-156, Jan. 2011.

[32] M. García-Vigueras, J. L. Gómez-Tornero, G. Goussetis, A. R Weily, and Y. J. Guo, "Enhancing frequency-scanning response of leaky-wave antennas using high-impedance surfaces," IEEE Antennas Wireless Propag. Lett., vol. 10, pp. 7-10, 2011.

[33] M. García-Vigueras, J. L. Gómez-Tornero, G. Goussetis, A. R. Weily, and Y. J. Guo, "1D Leaky wave antenna employing parallel-plate waveguide loaded with PRS and HIS," IEEE Trans. Antennas Propag., vol. 59, no. 10, pp. 3687-3694, Oct. 2011.

[34] G. Goussetis, A. P. Feresidis, and J. C. Vardaxoglou, "Tailoring the AMC and EBG characteristics of periodic metallic arrays printed on grounded dielectric substrate," IEEE Trans. Antennas Propag., vol. 54, no. 1, pp. 82-89, Jan. 2006.

[35] Y. R. Lee, A. Charaya, D. S. Lockyer, and J. C. Vardaxoglou, "Dipole and tripole metallodielectric photonic bandgap (MPBG) structures for microwave filter and antenna applications," Inst. Electr. Eng. Proc. Optoelectron., vol. 127, pp. 395-400, Dec. 2000.

[36] M. Caiazzo, S. Maci, and N. Engheta, "A metamaterial surface for compact cavity resonators," IEEE Antennas Wireless Propag. Lett., vol. 3 , pp. 261-264, 2004

[37] V. Lomakin and E. Michielssen, "Beam transmission through periodic subwavelength hole structures," IEEE Trans. Antennas Propag., vol. 55 , no. 6, pp. $1564-1581$, Jun. 2007.

[38] M. García-Vigueras, J. L. Gómez-Tornero, G. Goussetis, J. S. GómezDiaz, and A. Álvarez-Melcón, "A modified pole-zero technique for the synthesis of waveguide leaky-wave antennas loaded with dipole-based FSS," IEEE Trans. Antennas Propag., vol. 58, no. 6, pp. 1971-1979, Jun. 2010.

[39] S. A. Tretyakov, Analytical Modeling in Applied Electromagnetics. Norwood, MA: Artech House, 2003.

[40] J. B. Pendry, A. J. Holden, W. J. Stewart, and I. Youngs, "Extremely low frequency plasmons in metallic mesostructures," Phys. Rev. Lett., vol. 76, no. 25, pp. 4773-4776, Jun. 1996.

[41] Y. Zhao, P. A. Belov, and Y. Hao, "Modelling of wave propagation in wire media using spatially dispersive finite-difference time-domain method: Numerical aspects," IEEE Trans. Antennas Propag., vol. 55, no. 6, pp. 1506-1513, Jun. 2007.
[42] J. Brown, "Artificial dielectrics having refractive indices less than unity," Inst. Electr. Eng. Proc. IV-Inst. Monographs, vol. 100, no. 62R, pp. 51-62, Oct. 1953.

[43] J. W. Miles, "The equivalent circuit for a plane discontinuity in a cylindrical waveguide," Proc. IRE, vol. 34, pp. 728-742, Oct. 1946.

[44] N. Marcuvitz, Waveguide Handbook, ser. MIT Radiation Laboratory Series, New ed. New York: McGraw-Hill, 1951, vol. 10.

[45] A. Wexler, "Solution of waveguide discontinuities by modal analysis," IEEE Trans. Microw. Theory Tech., vol. MTT-15, no. 9, pp. 508-517, Sep. 1967

[46] F. Medina, F. Mesa, and R. Marqués, "Extraordinary transmission through arrays of electrically small holes from a circuit theory perspective," IEEE Trans. Microw. Theory Tech., vol. 56, pp. 3108-3120, Dec. 2008

[47] R. Rodríguez-Berral, F. Mesa, and F. Medina, "Circuit model for a periodic array of slits sandwiched between two dielectric slabs," Appl. Phys. Lett., vol. 96, no. 16, p. 161104(1-3), 2010.

[48] M. Beruete, M. Navarro-Cía, S. A. Kuznetsov, and M. Sorolla, "Circuit approach to the minimal configuration of terahertz anomalous extraordinary transmission," Appl. Phys. Lett., vol. 98, no. 1, p. 014106(1-3), 2011.

[49] M. Beruete, M. Navarro-Cía, and M. Sorolla, "Understanding anomalous extraordinary transmission from equivalent circuit and grounded slab concepts," IEEE Trans. Microw. Theory Tech., vol. 59, no. 9, pp. 2180-2188, Sep. 2011.

[50] R. Rodríguez-Berral, F. Medina, F. Mesa, and M. García-Vigueras, "Quasi-analytical modeling of transmission/reflection in strip/slit gratings loaded with dielectric slabs," IEEE Trans. Microw. Theory Tech., vol. 60, no. 3, pp. 405-418, Mar. 2012

[51] M. García-Vigueras, F. Mesa, F. Medina, R. Rodríguez-Berral, and J. L. Gómez-Tornero, "Equivalent circuits for conventional and extraordinary reflection in dipole arrays," in Proc. Int. Microw. Symp., Baltimore, MD, Jun. 2011, DOI: 10.1109/MWSYM.2011.5972674.

[52] A. K. Bhattacharyya, Phased Array Antennas Floquet Analysis, Synthesis, BFNs, and Active Array Systems. Hoboken, NJ: Wiley, 2006.

[53] D. M. Pozar, Microwave Engineering, 3rd ed. Hoboken, NJ: Wiley, 2005.

[54] U. Fano, "Effects of configuration interaction on intensities and phase shifts," Phys. Rev. Lett., vol. 124, pp. 1866-1878, 1961.

[55] E. A. Parker and S. M. A. Hamdy, "Rings as elements for frequency selective surfaces," Electron. Lett., vol. 17, no. 17, pp. 612-614, Aug. 1981.

[56] S. A. Hosseini, F. Capolino, and F. De Flaviis, "A 44 GHz single-feed Fabry-Pérot cavity antenna designed and fabricated on quartz," in Proc. IEEE Int. Symp. Antennas Propag., Jul. 2011, pp. 1285-1288.

[57] G. I. Kiani, L. G. Olsson, A. Karlsson, K. P. Esselle, and M. Nilsson, "Cross-dipole bandpass frequency selective surface for energy-saving glass used in buildings," IEEE Trans. Antennas Propag., vol. 59, no. 2, pp. 520-525, Feb. 2011.

[58] F. C. Seman, R. Cahill, V. F. Fusco, and G. Goussetis, "Design of a salisbury screen absorber using frequency selective surfaces to improve bandwidth and angular stability performance," IET Microw. Antennas Propag., vol. 5, no. 2, pp. 149-156, Jan. 2011.

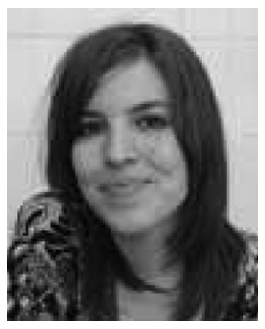

María García-Vigueras (S’09) was born in Murcia, Spain, in 1984. She received the Telecommunications Engineer degree from the Universidad Politécnica de Cartagena (UPCT), Murcia, Spain, in 2007, where she is currently working toward the Ph.D. degree in electrical engineering.

In 2008, she joined the Department of Communication and Information Technologies, UPCT, as a Research Assistant. She has been a visiting Ph.D. student at Heriot-Watt University, Edinburgh, Scotland, U.K.; the University of Seville, Seville, Spain; and the Queen's University, Belfast, Northern Ireland, U.K. Her research interests focus on the development of equivalent circuits to characterize periodic surfaces, with application to the analysis and design of leaky-wave antennas. 


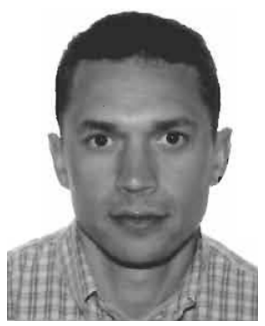

Francisco Mesa (M'93-SM’11) was born in Cádiz, Spain, in April 1965. He received the Licenciado and Doctor degrees in physics from the Universidad de Sevilla, Seville, Spain, in 1989 and 1991, respectively.

He is currently a Professor in the Department of Applied Physics, University of Seville. His research interests focus on electromagnetic propagation/radiation in planar structures.

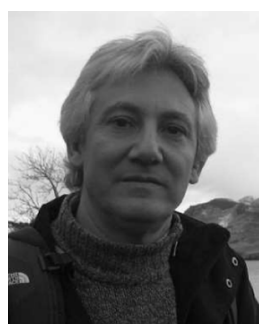

Francisco Medina (M'90-SM'01-F'10) was born in Puerto Real, Cádiz, Spain, in November 1960. He received the Licenciado and Doctor degrees in physics from the University of Seville, Seville, Spain, in 1983 and 1987, respectively.

From 1986 to 1987, he worked at the Laboratoire de Microondes de l'ENSEEIHT, Toulouse, France, as the recipient of a Spanish Ministry of Science and Education Research Scholarship. From 1985 to 1989 , he was an Assistant Professor with the Department of Electronics and Electromagnetism, University of Seville, where, since 1990, he has been an Associate Professor of Electromagnetism. He is currently a Full Professor of Electromagnetism (since July 2009) and Head of the Microwaves Group. His research interest includes analytical and numerical methods for guiding, resonant, and radiating structures, passive planar circuits, periodic structures, and the influence of anisotropic materials (including microwave ferrites) on such systems. He is also interested in artificial media modeling, periodic structures, and extraordinary transmission phenomena.

Dr. Medina is on the Editorial Board of the International Journal of $R F$ and Microwave Computer-Aided Engineering. He is a reviewer of the IEEE TRANSACTIONS ON MICROWAVE THEORY AND TECHNIQUES and of many other IEEE, Institution of Electrical Engineers (IEE), U.K., and American Physics Society journals. He has been a member of the Technical Programme Committees (TPC) of several major international and local conferences and has organized a few conferences and workshops. He is a Fellow of the Massachusetts Institute of Technology (MIT) Electromagnetics Academy.

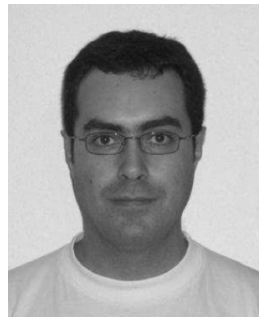

Raúl Rodríguez-Berral was born in Casariche (Seville), Spain, in 1978. He received the Licenciado (M.Sc.) and Ph.D. degrees in physics from the University of Seville, Seville, Spain, in 2001 and 2008, respectively.

In January 2002, he joined the Department of Applied Physics, University of Seville, where he is currently an Assistant Professor. His research interests include the study of the spectrum and the excitation of periodic and nonperiodic planar structures and highfrequency circuit modeling.

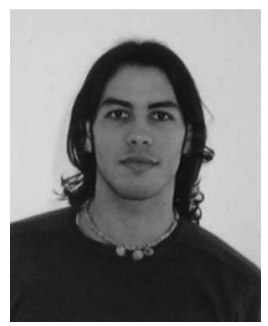

José L. Gómez-Tornero (M’06) was born in Murcia, Spain, in 1977. He received the Telecommunications Engineer degree from the Polytechnic University of Valencia (UPV), Valencia, Spain, in 2001, and the $\mathrm{Ph} . \mathrm{D}$. degree (laurea cum laude) in telecommunication engineering from the Technical University of Cartagena (UPCT), Cartagena, Spain, in 2005.

In 1999, he joined the Radio Communications Department, UPV, as a research student, where he was involved in the development of analytical and numerical tools for the automated design of microwave filters in waveguide technology for space applications. In 2000, he joined the Radio Frequency Division, Industry Alcatel Espacio, Madrid, Spain, where he was involved with the development of microwave active circuits for telemetry, tracking, and control (TTC) transponders for space applications. In 2001, he joined the UPCT as an Assistant Professor. From October 2005 to February 2009, he held de position of Vice Dean for Students and Lectures affairs in the Telecommunication Engineering Faculty at the UPCT. Since 2008, he has been an Associate Professor at the Department of Communication and Information Technologies, UPCT. His current research interests include analysis and design of leaky-wave antennas and the development of numerical methods for the analysis of novel passive radiating structures in planar and waveguide technologies.

Dr. Gómez-Tornero received in July 2004 the national award from the foundation EPSON-Ibérica to the best Ph.D. project in the field of Technology of Information and Communications (TIC). In June 2006, he received the Vodafone foundation-COIT/AEIT (Colegio Oficial de Ingenieros de Telecomunicación) award to the best Spanish Ph.D. dissertation in the area of advanced mobile communications technologies. This dissertation was also awarded in December 2006 as the best dissertation in the area of electrical engineering, by the Technical University of Cartagena. In February 2010, he was appointed CSIRO Distinguished Visiting Scientist by the CSIRO ICT Centre, Sydney, Australia. 